\title{
Triadi di suonatrici nella Sicilia e nella Calabria di età greca (IV-III sec. a.C.)
}

\author{
Angela BELLIA*
}

RÉSUMÉ Triades de musiciennes en Sicile et Calabre d'époque grecque (IV - III s. a.C.) Parmi les terres cuites figurées à représentation musicale se distinguent en Sicile des groupes de trois musiciennes. Si le premier exemple, de Gela, date du V ${ }^{\mathrm{e}}$ s. av. J.-C., c'est surtout à l'époque hellénistique que différentes versions en ont été diffusées. Une joueuse d'aulos est toujours présente au centre ou sur les côtés, tandis que les autres musiciennes tiennent un tympanon et parfois des cymbales. Cette composition n'est connue en dehors de Sicile qu'à Locres, où ont été recueillis des exemplaires datés fin du $\mathrm{IV}^{\mathrm{e}}$ - début du III ${ }^{\mathrm{e}}$ s. av. J.-C. - Sur 68 triades, 27 proviennent d'aires sacrées où est attesté un culte lié à l'eau, ce qui a suggéré l'identification des musiciennes avec des Nymphes. En Sicile, le culte de celles-ci est étroitement lié à celui d'Artémis, mais cela n'exclut pas un lien éventuel avec un rituel adressé aux divinités chthoniennes. Musiciennes, les Nymphes pourraient ainsi jouer un rôle important dans les fêtes en l'honneur d'Artémis et à l'occasion du mariage de Korè-Perséphone. - Les questions soulevées ici réclameront à l'avenir un examen approfondi et interdisciplinaire. La signification de l'offrande des ces statuettes ainsi que leur lien avec la musique sont problématiques, tout comme leur identification : elles peuvent représenter des déesses ou des mortelles, prêtresses ou simples dédicantes.

MOTS CLEFS Musiciennes, Nymphes, Artémis, Korè-Perséphone, Sicile, triade.

ABSTRACT Triads of Musicians in Sicily and Calabria during the Greek Period (4 $4^{\text {th }}$ and $3^{\text {rd }}$ c. B.C.) Within the coroplastics with musical representations, there is in Sicily a category of groups of three female musicians. The first example comes from Gela and dates back to the $5^{\text {th }}$ c. B.C. but different versions of the triad are more diffused above all in the Hellenistic age. The presence of aulos players is constant in the centre or at the sides while the other figures hold the tympanon and in some cases the kymbala. We do not know of any other examples outside Sicily, except for findings from Locri dated end of the $4^{\text {th }}$ - beginning of the $3^{\text {rd }}$ c. B.C. - Of the 68 female triads, 27 were found in sacred areas where there is documentary evidence for a cult connected with water. Such a circumstance prompted the identification of the musicians with the Nymphs. In Sicily, their cult seems closely connected to Artemis. But it does not exclude that these particular representations might be bound with a sacred ritual to cthonic divinities. The Nymphs, in the role of musicians, could play a significant role in the festivities connected with Artemis and with the marriage celebrations of Kore-Persephone. Numerous questions have been raised that will require deeper, interdisciplinary, study in the future. There is a problem of understanding the meaning of the dedication of these terracottas and their relationship to music. Closely connected to this is the theme of the identification of some typologies of the female triads that could represent divinities, priestesses or simply offerers.

KEY WORDS Female musicians, Nymphs, Artemis, Kore-Persephone, Sicily, triad.

* Università di Bologna, Italia 
Angela Bellia, Triadi di suonatrici nella Sicilia e nella Calabria di età greca (IV-III sec. a.C.), in (éd.) S. HuYSECOM-HAXHI, A. Muller, Figurines grecques en contexte. Présence muette dans le sanctuaire, la tombe et la maison, Lille, Presses universitaires du Septentrion-Université de Lille 3, 2015, pp. 107-126.

La coroplastica con raffigurazioni musicali costituisce un campo privilegiato di indagine per comprendere la funzione della musica nel suo contesto di produzione e fruizione. Lo studio di queste particolari terrecotte, emblemi e testimoni dello stretto legame tra la pratica musicale e l'ambito sacro, documenta luoghi e aspetti degli eventi sonori legati ai riti nel mondo antico e aiuta a ricostruire sia il ruolo degli strumenti musicali nella sfera religiosa sia il significato della musica per le società antiche. Il lavoro di ricerca sistematico della coroplastica con raffigurazioni musicali è stato avviato recentemente ${ }^{1}$ : l'analisi ha consentito di individuare, tra gli altri, il «gruppo» delle triadi di figure femminili che reggono o suonano strumenti musicali, la cui presenza è ampiamente documentata in Sicilia e in Calabria (fig. 1) ${ }^{2}$. Si tratta di terrecotte che, sulla base dei contesti di rinvenimento, sono state datate dagli archeologi nel periodo compreso tra la fine del V e l'inizio del III sec. a.C. ${ }^{3}$. Le statuette, che misurano tra 8 e $13 \mathrm{~cm}$ di alt. e 12 e $15 \mathrm{~cm}$ in largh., sono state trovate in Sicilia ad Adrano (fig. 2) ${ }^{4}$, ad Agrigento (fig. 3) ${ }^{5}$, a Palazzolo Acreide (fig. 4) ${ }^{6}$, a Ciminna (fig. 5) ${ }^{7}$, a Gela (fig. 6) ${ }^{8}$ a Castel di Tusa (fig. 7) ${ }^{9}$, a Monte Iato (fig. 8) ${ }^{10}$, a Monte Saraceno (fig. 9) ${ }^{11}$, a Lentini (fig. 10) ${ }^{12}$, a

\section{BELLIA 2009.}

2 Allo stato degli studi, una sola teoria fittile, composta da tre figure femminili con alto polos sedute dietro una trapeza (prima metà del VI sec. a.C.), è stata trovata a Taranto. Si veda ABRUZZESE CALABRESE 1996, p. 191, fig. 132.

3 Il primo esempio proviene da Gela. ORLANDINI 1960, p. 204-205, n. 4; BelLia 2009, p. 47, n. 53.

4 LAMAgNa 1994, p. 78-79, tav. XLIX, fig. 1, 4; Bellia 2009, p. 23, n. 4-7; p. 25-26, n. 13-16. Tre triadi sono state trovate in un contesto riferibile ad ambito cultuale, probabilmente un deposito o uno scarico connesso all'attività di una bottega artigiana legata ad un santuario. In via Catania, probabilmente area dell'abitato antico, sono stati trovati altri tre esemplari. Si segnala anche un rinvenimento sporadico. Si veda BELLIA 2009, p. 22-26.

5 Winter 1903, II, p. 140, fig. 8; BReitenstein 1945, p. 135-136, fig. 51; BELlia 2009, p. 32-33, n. 28-30. Tre gruppi di suonatrici provengono dal «santuario rupestre», collocato sulle pendici della Rupe Atenea, nell'acropoli della città greca.

6 Bernabò Brea 1956, tav. XXXII, fig. 6; Bellia 2009, p. 35, n. 34. Si tratta di un rinvenimento sporadico.

7 PACE 1945, p. 623, fig. 166; Bellia 2009, p. 36, n. 36. Dell'esemplare non si conosce la provenienza.

8 ORLANDini 1968, p. 58, fig. 41; PANVINI 2000, p. 30, n. 31-33; Bellia 2005, p. 61-62, figg. 10-11; BELLIA 2009, p. 42-51, n. 45; 52-53; 59; 62-64. Una triade è stata trovata in una abitazione di età ellenistica nell'area urbana di Capo Soprano; un frammento proviene dall'acropoli della città occupata da un settore abitativo; un altro dalla fattoria-officina di Manfria; tre esemplari sono di provenienza incerta.

9 CAREtToni 1959, p. 314, fig. 22 a-b; Bellia 2009, p. 52, n. 65-66. I due frammenti di triade erano in uno scarico.

10 ISLER 1992, p. 7, 9, fig. 3; BeLliA 2009, p. 54, n. 68. L'esemplare proviene dal teatro.

11 Siracusano 1996, p. 29, tav. XLII, 4; Sardella-Vanaria 2000, p. 100; Bellia 2007, p. 93-95, n. 77. Il frammento è stato trovato in una costruzione dell'Acropoli a destinazione cultuale.

12 FRASCA 1995, p. 10, n. 13, tav. II, c-d; BELLIA 2009, p. 61-63, n. 77; 81-83. Un gruppo di suonatrici proviene dall'area sacra di Colle San Mauro; due frammenti sono stati trovati nel complesso rupestre, forse a carattere cultuale, di Contrada Crocifisso; un altro nel pozzo dell'insediamento rupestre in località Caracausi. 
Angela Bellia, Triadi di suonatrici nella Sicilia e nella Calabria di età greca (IV-III sec. a.C.), in (éd.) S. HuYSECOM-HAXHI, A. Muller, Figurines grecques en contexte. Présence muette dans le sanctuaire, la tombe et la maison, Lille, Presses universitaires du Septentrion-Université de Lille 3, 2015, pp. 107-126.

Morgantina (fig. 11) $)^{13}$, a Butera (fig. 12) (4 $^{14}$ a Scornavacche (fig. 13) ) $^{15}$ a Segesta $\left(\text { fig. 14) }{ }^{16} \text {, a Selinunte (fig. 15) }\right)^{17}$, a Solunto (fig. 16) ${ }^{18}$ e a Siracusa (fig. 17) $)^{19}$. In Calabria, le triadi di suonatrici sono presenti a Reggio Calabria (fig. 18) ${ }^{20}$ e a Locri (fig. 19) ${ }^{21}$, dove sono stati trovati anche i gruppi di quattro (fig. 20) ${ }^{22}$ o cinque figure femminili (fig. 21) ${ }^{23}$, alcune delle quali con strumenti musicali a fiato e a corde. Tre (fig. 22) ${ }^{24}$ o quattro (fig. 23) ${ }^{25}$ personaggi femminili danzanti e con strumenti musicali a fiato e a percussione, talvolta posti dietro l'altare, sono nelle scene rituali dei pinakes trovati a Lipari che, per tecnica e raffigurazioni, si distinguono dalle triadi fittili che sono fornite di retro ${ }^{26}$. Analoghe tavolette in frammenti sono state trovate a Messina ${ }^{27} \mathrm{e}$ a Salina ${ }^{28}$.

Trentotto triadi trovate in Sicilia provengono da aree sacre, diciassette da abitato e da botteghe artigianali e due da necropoli; gli esemplari rinvenuti in Calabria provengono

13 Bell 1981, p. 162-163, tavv. 62-63; BELL 1987, p. 117, tav. XXXVIII, fig. 2; WILSON 2000, p. 11; RAFFIOTTA 2007, p. 107, n. 151, fig. 29; RAFFIOTTA 2009, 121-122, fig. 34; BELlia 2009, p. 99-105, n. $210-215 ; 217-230 ; 232-240 ; 244-246$. Triadi, talvolta in frammenti, sono state trovate a nell'Agorà di Morgantina nel Theater foundation fill. I $\mathrm{C}_{2}$ (una triade); nel Lower sector. I B (un frammento); nel Naiskos. I D (un esemplare); nel West Shops, destruction fill. I F2 (un frammento); nel Kiln. I N (una triade). Altri esemplari erano nella Fontana ellenistica (una triade); in Contrada Vinci. II G (un esemplare); nella Necropoli III, fill (un frammento); nella Necropoli III. Epitymbion VII (due esemplari); nella Papa Hill. VI A (una triade); nel North Sanctuary. Court C, cistern (un esemplare); nel North Sanctuary (tre triadi); nel Santuario Francesco Bisconti (un esemplare).

14 AdAMESTEANU 1958a, col. 639, fig. 260; ADAMESTEANU 1958b, tav. V, fig. 8b; PANVINI 1998, p. 247; GuzZone 2003, p. 127, fig. 10; Portale 2008, fig. 21a; Bellia 2009, p. 99-105, n. 315-319. Nel santuario di Fontana Calda sono state trovate cinque triadi, due delle quali frammentarie.

15 Bellia 2009, p. 132, n. 332. Un esemplare proviene dall'abitato che attiene ad un villaggio di coroplasti e ceramisti.

16 DE Cesare 2008, p. 637; 639, n. 11, tav. CVI; Bellia 2009, p. 133, n. 334. Si segnala un solo frammento.

17 KeKULÉ 1884, p. 14, fig. 20; Bellia 2009, p. 137, n. 343. Si tratta di un rinvenimento sporadico.

18 BELLIA 2009, p. 138, n. 344. L'esemplare è stato trovato in un'area, forse sede di culto.

19 ORSI 1900, p. 383, fig. 29; BELLIA 2009, p. 143, n. 355. Un frammento è stato trovato nell'Artemision di Scala Greca.

20 Inedita. Museo Archeologico Nazionale di Reggio Calabria. Inv. 359. Un esemplare frammentario è stato trovato nel Santuario di Griso Laboccetta.

21 Tropea 1991, p. 180-185, n. 1-8, figg. 286-293; BELlia 2012a, p. 109-119, figg. 25-37. Otto esemplari sono stati trovati nel santuario rupestre di Grotta Caruso.

22 TROPEA 1991, p. 182, n. 9-10, fig. 294; BELliA 2012a, p. 113, fig. 30. Due gruppi di suonatrici sono stati trovati nel santuario rupestre di Grotta Caruso.

23 BELlia 2012a, p. 118, fig. 36. Un solo esemplare proviene dal santuario rupestre di Grotta Caruso.

24 SARDELla-VANARIA 2000, p. 95-97, tavv. I-IX; BELlia 2009, p. 70-84, n. 100-159; Bellia 20062010, p. 15-16, figg. 3-15. Le tavolette sono state trovate nel santuario dell'ex Proprietà Maggiore.

25 SARDElla-VANARIA 2000, p. 94-102, tav. X; Bellia 2009, p. 70-86, n. 160-166; Bellia 2006-2010, p. 17, figg. 16-18. Anche i pinakes con quattro figure provengono dal santuario dell'ex Proprietà Maggiore.

26 E' un gruppo molto numeroso, costituito da ben 3325 esemplari, ordinati in «tre schemi figurativi» principali e quattordici «tipi» diversi che possono presentare «varianti».

27 SCIBONA 1999, p. 59, VSM/12; p. 64-65, VSM/46-47. I frammenti sono stati trovati nei pressi della necropoli.

28 VANARIA 2000, p. 69, tav. 1, n. 1. Il frammento proviene dall'area sacra extraurbana nei pressi del Santuario della Madonna del Terzito in località Valdichiesa di Leni. 
Angela Bellia, Triadi di suonatrici nella Sicilia e nella Calabria di età greca (IV-III sec. a.C.), in (éd.) S. HuYSECOM-HAXHI, A. Muller, Figurines grecques en contexte. Présence muette dans le sanctuaire, la tombe et la maison, Lille, Presses universitaires du Septentrion-Université de Lille 3, 2015, pp. 107-126.

da contesti $\operatorname{sacri}^{29}$. Le tre figure femminili sono disposte l'una accanto all'altra. Il loro numero è probabilmente idoneo a rappresentare un adeguato gruppo di partecipanti e a esprimere la pluralità ${ }^{30}$ nell'ambito di attività corali e musicali con l'aulos ${ }^{31}$, il tympanon $^{32}$ e i kymbala ${ }^{33}$.

Le suonatrici di aulos tengono lo strumento al petto con entrambe le mani (fig. 24); possono essere poste a sinistra (fig. 25) o al centro (fig. 26); portano orecchini di forma globulare (fig. 27) e capelli raccolti in alto a crocchia sul capo che potrebbe connotare la giovane età delle figure (fig. 28) ${ }^{34}$; talvolta indossano anche un copricapo conico e a punta (fig. 6) o il polos (fig. 10). Una delle auletrie con questo copricapo, forse indizio di uno status sacro del personaggio ${ }^{35}$, è raffigurata all'interno di un'edicola dotata di frontoncino: si tratta forse della rappresentazione di uno spazio religioso adibito alle esecuzioni musicali (fig. 8) ${ }^{36}$.

Le suonatrici di tympanon suonano lo strumento con la mano destra (fig. 29) o lo reggono con quella sinistra accostandolo al busto (fig. 25); talvolta indossano un copricapo a punta (fig. 26) o hanno complicate acconciature (fig. 2); più frequentemente portano un polos cilindrico sul capo (fig. 30); in qualche caso sollevano con la mano destra un lembo dell'abito trasparente (fig. 12) ${ }^{37}$. Dove si conserva, il colore usato per il tympanon è il rosso (fig. 31).

Le suonatrici di kymbala, che indossano un basso copricapo, percuotono le due parti dello strumento l'una contro l'altra accostandole al ventre (fig. 32). Alcune figure, che

29 Si veda BELLIA 2009, p. 168-170.

30 HinZ 1998, p. 48, nota 313. Cfr. BURKERT 2003, p. 339; SALAPATA 2009, p. 332.

31 Per l'aulos, cfr. BAINES 1995, p. 235-237; SACHS 1996, p. 156-159; SACHS-HORNBOSTEL 2002, p. 460-461; WEST 2007, p. 129-164. Per la raffigurazione dello strumento nella ceramica attica, si vedano almeno PAQUETTE 1984, p. 23-61; BUNDRICK 2005, p. 34-42. Per il rinvenimento di auloi in Sicilia e nell'Italia meridionale, si veda BELLIA 2010, p. 103-108; BELLIA 2012b, p. 91-109.

32 Per questo strumento musicale, cfr. SACHS 1996, p. 170-171; SACHS-HORNBOSTEL 2002, p. 435; WEST 2007, p. 192-194. Per la raffigurazione dello strumento nella ceramica attica, si vedano almeno PAQUETTE 1984, p. 206; BUNDRICK 2005, p. 48. Per l'iconografia del tympanon nella ceramica italiota e siceliota, cfr. GUIZZI-STAITI 1992-1995, p. 59; JACQUET RIMASSA 1999, p. 53-58; DE CESARE 2005, p. 58-59. Piccoli tympana in bronzo del VI sec. a.C. sono stati trovati in Sicilia. BELLIA 2010, p. 9495; BELLIA 2012b, p. 43-49.

33 Per i kymbala, cfr. SACHS 1996, p. 171-172; WeSt 2007, p. 194; SACHS-HORNBOSTEL 2002, p. 420421. Si veda anche SCHATKIN 1978, p. 147-172. Per la raffigurazione dei kymbala nella ceramica attica e italiota, si vedano PAQUETTE 1984, p. 206; BUNDRICK 2005, p. 47-48. Per i kymbala in bronzo dall'età del Ferro all'età ellenistica in Sicilia e in Calabria, cfr. BELLIA 2010, p. 87-90; BELLIA 2012b, p. 3-14.

34 L'acconciatura ad alta crocchia potrebbe essere un elemento della 'condizione virginale' delle figure. Cfr. Portale 2008, p. 11.

35 In generale per tale copricapo MüLLER 1915. Per il polos come elemento identificativo della divinità, cfr. BELL 1977, p. 142-143; BELL 1981, p. 81-82. Il particolare copricapo è posto sulla testa di figure, come eroi, sacerdoti, sacerdotesse e offerenti, cfr. ALBERTOCCHI 2004, p. 158; BARRA BAGNASCO 1986, p. 120, nota 25; CIPRIANI 1989, p. 143; SARDELLA-VANARIA 2000, p. 97, nota 26 e p. 111, nota 122; SguaitamatTi 1984, p. 53-54.

36 Per la presenza di una figura femminili che suona l'aulos all'interno di un tempio di stile misto, ionico e dorico, nei pinakes di Locri, cfr. Pinakes di Locri II, p. 90-97, n. 1-5; BELliA 2012a, p. 43-46, fig. 11.

37 Per la trasparenza degli indumenti delle suonatrici che potrebbero alludere ad una sessualità che sta per svelarsi, cfr. PORTALE 2008, p. 42. 
Angela Bellia, Triadi di suonatrici nella Sicilia e nella Calabria di età greca (IV-III sec. a.C.), in (éd.) S. HuYSECOM-HAXHI, A. Muller, Figurines grecques en contexte. Présence muette dans le sanctuaire, la tombe et la maison, Lille, Presses universitaires du Septentrion-Université de Lille 3, 2015, pp. 107-126.

non hanno strumenti musicali, con un lembo del chitone sollevato, sono in atteggiamento di danza (fig. 33) ${ }^{38}$, altre, senza particolari attributi, che sembrerebbero avere le labbra dischiuse (fig. 34) ${ }^{39}$, sono verosimilmente le cantatrici.

La prima documentazione di questi particolari ex-voto risale alla monumentale trattazione delle terrecotte di R. Kekulé e F. Winter, che dedicarono un certo spazio alle statuette di suonatrici di strumenti musicali ${ }^{40}$. Anche Orsi inserì qualche breve cenno a proposito dei frammenti di triadi rinvenuti nell'Artemision di Scala Greca ${ }^{41}$. Per primo il Pace aveva suggerito di riconoscere le Ninfe nella triade rinvenuta a Ciminna ${ }^{42}$. La stessa interpretazione è stata attribuita ai gruppi di suonatrici rinvenuti da Adamesteanu a Butera ${ }^{43}$ e da Orlandini a Gela ${ }^{44}$, e successivamente anche altri hanno adottato tale proposta $^{45}$. L'ipotesi è stata sostenuta dal rinvenimento dei fittili in luoghi sacri in cui è documentata anche un'attività cultuale in relazione alle acque. Le triadi di suonatrici, Ninfe o semplici offerenti ${ }^{46}$, potrebbero essere legate sia all'ambito sacro di Artemide, sia a quello di Persefone e ai diversi momenti delle feste che celebravano le nozze della $\operatorname{dea}^{47}$.

Un passo di Timeo ${ }^{48}$ riportato da Ateneo ci offre la testimonianza di una festa per le Ninfe in Sicilia: venivano offerti sacrifici ed esse erano oggetto di festeggiamenti da parte dei cittadini che ebbri si spostavano di casa in casa, vegliando tutta la notte, ballando, e probabilmente suonando, intorno alle statue in una specie di sospensione rituale dell'ordine. Il culto delle Ninfe, termine che indica "sia gli esseri divini, che governano su fiori e sorgenti, come pure le spose, le giovani donne al primo incontro amoroso e le giovani donne in età da marito» ${ }^{49}$, è legato ai riti di passaggio e di iniziazione al matrimonio e al ruolo di danzatrici delle fanciulle durante i riti in onore di

38 Per il legame tra la danza la sfera nuziale e giovanile con il gesto di sollevare l'abito, si veda MERKER 2000, p. 117.

39 BELLIA 2009, p. 26, n. 15.

40 KeKULÉ 1884, tavv. XIII, XVI; WINTER 1903, I, p. 53-56, 216, 227-228, 257; WINTER 1903, II, p. 138143.

41 ORSI 1900, p. 353-387.

42 PACE 1945, p. 623.

43 ADAMESTEANU 1958a, col. 639.

44 ORLANDINI 1968, p. 56;

45 Bell 1981, p. 92-93; MERTENS HORN 1991, p. 21-22; TROPEA 1991, p. 184-185; ISLER 1992, p. 7; FRASCA 1995, p. 10-13; LAMAGNA 1997-1998, p. 78-79; LARSON 2001, p. 220-221; ZOPPI 2004, p. 77; PORTALE 2008, p. 26-29.

46 Per il culto delle Ninfe in Sicilia in relazione con Demetra e Core/Persefone e Artemide, cfr. CIACERI 2004, p. 137-145; per il culto delle Ninfe in Sicilia, cfr. ANGELINI 1994, p. 25-42; LAGONA 1962, p. 33-35; MERTENS HORN 1991, p. 9-28. Per il rapporto tra le Ninfe e la musica, cfr. KOLLER 1995, p. 104-107. Per il rinvenimento di frammenti di aulos nei luoghi di culto dedicati alle Ninfe, cfr. BÉLIS 1984, p. 176-181. Si veda inoltre AMANDRY 1984, p. 395-425.

47 Il culto di Artemide a Siracusa era in relazione e quello per Demetra e Core. Secondo Diodoro Siculo, Biblioteca Storica V 3, 4, Artemide avrebbe ricevuto in dono dalle due dee l'isola di Ortigia. Per Pausania VIII 37, 6, Artemide era figlia di Demetra, mentre Cicerone, Sulla natura degli dei III 23, la chiama figlia di Persefone con la quale è anche identificata (Scolî a Teocrito XI, 12). Artemide e Atena sono le compagne di giochi di Core e con lei raccolgono fiori in un prato prima del suo rapimento (Inno a Demetra v. 424).

48 Timeo, FGrHist 566 F 32, in Ateneo, I Deipnosofisti VI 250a.

49 BURKERT 2003, p. 300. 
Angela Bellia, Triadi di suonatrici nella Sicilia e nella Calabria di età greca (IV-III sec. a.C.), in (éd.) S. HuYSECOM-HAXHI, A. Muller, Figurines grecques en contexte. Présence muette dans le sanctuaire, la tombe et la maison, Lille, Presses universitaires du Septentrion-Université de Lille 3, 2015, pp. 107-126.

divinità che presiedevano le nozze. Tra queste, Artemide era festeggiata dalle ragazze che, come le Ninfe, danzavano in cerchio ${ }^{50}$.

La relazione fra la musica e la sfera sacra della divinità in Sicilia, ed in particolare a Siracusa, è ricordata dalle fonti scritte. Secondo una testimonianza di Teocrito ${ }^{51}$, la presenza di Artemide era annunciata nella città dal suono di strumenti a percussione. Ateneo informa che a Siracusa era praticata la «danza dei messaggeri» o la «danza di Artemide messaggera ${ }^{52}$. Il passo di Ateneo concorda con la notizia del siracusano Epicarmo che ricorda l'uso dell'aulos per la melodia dedicata ad Artemide Chitonea ${ }^{53}$. Non va trascurato che Ateneo definisce «ionica» la danza, probabilmente per sottolinearne il suo carattere licenzioso e orgiastico ${ }^{54}$. Il passo di Ateneo trova anche conferma nella notizia di Polluce che ricorda la pratica della danza in Sicilia nell'ambito religioso della dea $^{55}$. È significativo che la danza ionica ${ }^{56}$ venga citata in relazione sia con la danza per Artemide Chitonea ${ }^{57}$, sia con la «danza degli ubriachi» che, secondo Luciano $^{58}$, era di origine frigia, accompagnata dall'aulos ed eseguita da contadini ${ }^{59}$. Queste testimonianze sulla danza a Siracusa e l'uso dell'aulos per accompagnarla concordano con quanto è ricordato da Probo ${ }^{60}$ e Diomede ${ }^{61}$. I grammatici informano che nella città, durante la festa dedicata ad Artemide Lyaia, fosse consuetudine che pastori e contadini, arrivati dalla campagna, disputassero gare e si recassero ubriachi di casa in casa, cantando in onore della dea ${ }^{62}$. Lo speciale appellativo di Artemis Angelos ${ }^{63}$ con cui

50 CALAME 2001, p. 129-131. Si veda anche KOLLER 1995, p. 96-98. Per la danza corale in Sicilia, si veda WILSON 2007, p. 351-377.

51 Teocrito, Idilli II 36.

52 Ateneo, I Deipnosofisti XIV 629e.

53 Epicarmo, La Sfinge fr. 127 Kaibel. Il passo è riportato da Erodiano, III 1, 295, 3 e Stefano Bizantino, p. 694, 8; Amipsia, PCG II, p. 207, 21, 1; su cui vedansi LORENZ 1864, p. 252, n. 2; OLIVIERI 1921, p. 44, n. 74; RODRIGUEZ-GULLÉN 1996, p. 126-127, n. 199. Cfr. PORTALE 2008, p. 50.

54 PICKARD-CAMBRIDGE 1962, p. 257; SÉCHAN 1930, p. 154-156. Si veda inoltre TIBY 1993, p. 29-30.

55 Polluce, Onomasticon IV 103. Cfr. CIACERI 2004, p. 98, nota 8. Per la danza ionica, cfr. LAWLER 1943, p. 60-71; in particolare per la danza ionica in Sicilia, p. 67-68.

56 La danza ionica era in origine una danza cultuale caratterizzata da movimenti in cui gli esecutori reggevano in alto il chitone con una mano, mentre con l'altra portavano offerte, verosimilmente primizie per la divinità. Cfr. LAWER 1943, p. 60-71.

57 Per la connessione tra la danza ionica e la danza per Artemide Chitonea a Siracusa, cfr. LAWLER 1943, p. 68-69.

58 Luciano, Sulla danza 33, 33.

59 Trifone, Questioni di nomenclatura fr. 109 von Velsen, in Ateneo, I Deipnosofisti XIV 618c. Il boukolismos era una composizione per aulos accompagnata dalla danza. Si vedano PAPADOPOULOU 2004, p. 330, n. 266; PICKARD-CAMBRIDGE 1962, p. 155, n. 196; SIFAKIS 1971, p. 81-82.

60 Probo, In Vergilii Bucolica et Georgica III 1, ed. Thilo, p. 324-325.

61 Diomede, Artis Grammaticae III, in Grammatici latini ed. Keil, I, p. 486-487.

62 Per le feste siracusane in onore di Artemide eseguite al suono dell'aulos, cfr. CANFORA 2001, III, p. 1626, nota 1; PAPADOPOULOU 2004, p. 330, n. 265. Si veda anche REICHERT-SÜDBECK 2000, p. $75-$ 80 e PORTALE 2008, p. 50.

63 Cfr. CANFORA 2001, p. 1626, nota 3. Per le statuette di Artemide aposkousa, nel gesto forse in connessione con il ritorno di Persefone, di cui la dea sarebbe messaggera, si veda PORTALE 2008, p. 16. 
Angela Bellia, Triadi di suonatrici nella Sicilia e nella Calabria di età greca (IV-III sec. a.C.), in (éd.) S. HuYSECOM-HAXHI, A. Muller, Figurines grecques en contexte. Présence muette dans le sanctuaire, la tombe et la maison, Lille, Presses universitaires du Septentrion-Université de Lille 3, 2015, pp. 107-126.

la dea era venerata a Siracusa ${ }^{64}$ era forse in relazione con la festa che celebrava il ritorno di Core $^{65}$, verosimilmente comprendente l'esecuzione di danze e di musica ${ }^{66}$.

Il legame dell'aulos con la sfera rituale di Artemide $^{67}$ è confermato anche dal rinvenimento di questo strumento nell'area sacra del tempio ionico a Siracusa, dedicato alla dea ${ }^{68}$. Si tratta di sette sezioni di aulos in frammenti, databili al IV-III sec. a.C., forse pertinenti a diversi strumenti musicali ${ }^{69}$. Tre altri frammenti di aulos in osso del IV-III sec. a.C., uno dei quali presenta segni di rottura durante la lavorazione (in una bottega artigiana?), sono stati trovati ad Adrano ${ }^{70}$ nello stesso contesto delle suonatrici singole di aulos (fig. 35) e di tympanon (fig. 36) e delle triadi (figg. 25-28-34). ${ }^{71}$

Sebbene sia difficile stabilire con certezza se i coroplasti abbiano modellato intenzionalmente la misura delle canne dell'aulos, quello raffigurato con più frequenza nelle triadi è caratterizzato da corti tubi (fig. 37) che forse potrebbero appartenere agli auloi parthenioi, «tipo da fanciulla», caratterizzati da un timbro acuto e adatti a sovrastare la sonorità degli strumenti a percussione ${ }^{72}$. Con questi auloi, usati durante le cerimonie religiose che presentavano notevoli affinità con quelle nuziali ${ }^{73}$, venivano accompagnate le danze femminili, indispensabili ai riti di matrimonio ${ }^{74}$. La presenza dell'aulos, e talvolta degli strumenti a percussione, nella sfera nuziale è fornita dalle informazioni dei testi. Omero ${ }^{75}$ ricorda che lo strumento era usato per accompagnare il canto d'imeneo e la danza dei cortei che si svolgevano alla vigilia delle nozze. L'autore dello $\mathrm{Scudo}^{76}$, un poemetto attribuito ad Esiodo, ma forse risalente al 600-580 a.C., offre una rappresentazione della cerimonia nuziale e della musica adatta a fare baldoria e ad accompagnare il ritmo veloce della danza eseguita anche con l'aulos. I canti erano spesso eseguiti in coro dalle giovani compagne della sposa ${ }^{77}$ : un frammento di Saffo ${ }^{78}$ descrive il corteo per le nozze di Ettore e Andromaca e fornisce una ulteriore testimonianza dell'uso dell'aulos e degli strumenti a percussione per i canti delle fanciulle. Non mancano riferimenti alla musica eseguita con lo strumento per

64 Per gli appellativi di Artemide in Sicilia, cfr. CIACERI 2004, p. 97-102; NILSSON 1957, p. 187; PORTALE 2008, p. 14.

65 Si vedano NILSSON 1957, p. 356-362; BURKERT 2003, p. 317-318.

66 Glossarium Italicorum, n. 213, PCG I, p. 326.

67 Per gli aspetti musicali legati ad Artemide, cfr. ZsCHÄTZSCH 2002, p. 63-72. Si vedano anche BURKERT 2003, p. 297-302; CALAME 2011, p. 91-101 e p. 142-191.

68 DE CESARE 2001, p. 393, nota 26 con bibl. prec.

69 Bellia 2010, p. 103, fig. 44; BELlia 2012b, p. 94, fig. 92.

70 Bellia 2010, p. 103, fig. 45; BELLia 2012b, p. 97, fig. 94.

71 BELLIA 2009, p. 24-26.

72 Polluce, Onomasticon IV 81. Polluce precisa che gli «auloi con la voce da fanciulla» accompagnavano i canti corali di fanciulle.

73 GENTILI 1995, p. 117-151.

74 OAKLEY 2004, p. 312-314.

75 Omero, Iliade XVIII vv. 490-495. Cfr. MUTH 1954, p. 5-45; DEUSSEN 1973, p. 132; OAKLEY 2004, p. 313, n. 101; CALAME 2006, p. XVII.

76 Esiodo, Scudo vv. 272-285. Cfr. OAKLEY 2004, p. 313, n. 104.

77 CALAME 2006, p. XIX.

78 Saffo, fr. 44 Voigt, vv. 21-34. OAKLEY-SinOS 1993, p. 26. Potrebbe trattarsi di un epitalamio scritto da Saffo da eseguire in un cerimoniale rituale o in pubbliche occasioni. Cfr. PAGE 1955, p. 72; GENTILI 1995, p. 289-290. 
Angela Bellia, Triadi di suonatrici nella Sicilia e nella Calabria di età greca (IV-III sec. a.C.), in (éd.) S. HuYSECOM-HAXHI, A. Muller, Figurines grecques en contexte. Présence muette dans le sanctuaire, la tombe et la maison, Lille, Presses universitaires du Septentrion-Université de Lille 3, 2015, pp. 107-126.

accompagnare le parole di buon augurio rivolte agli $\operatorname{sposi}^{79}$ e i canti eseguiti davanti alla porta della camera nuziale ${ }^{80}$.

La notizia di Posidippo ${ }^{81}$, che ricorda una festa di matrimonio alla presenza delle sacerdotesse di Ade e Persefone, testimonia l'uso dell'aulos nel corso della veglia degli invitati ubriachi che durava tutta la notte. Questa testimonianza è interessante per la possibile presenza dello strumento durante le celebrazioni delle sacre nozze della coppia divina, la cui unione era, forse, considerata metafora di quella delle giovani donne ${ }^{82}$. I Theogamia erano feste solenni a ricorrenza annuale che comprendevano sacrifici e agoni ginnici ${ }^{83}$ : è probabile che vi trovasse spazio la sfera sonora.

L'esecuzione di danze, inscindibili dalla musica e dal canto, potrebbe avere avuto luogo nel contesto mitico rituale degli Anthesphoria ${ }^{84}$. Questo momento, forse fase iniziale dei Koreia, denominazione con cui in Sicilia venivano probabilmente indicate le molteplici feste in onore della dea $^{85}$, rievocava l'episodio del ratto di Core che avviava il dramma mitico $^{86}$. Il termine Anthesphoria è in relazione con Anthologia, l'azione di cogliere fiori cui Core era intenta al momento del rapimento ${ }^{87}$. Viene stabilito un rapporto tra la tradizione siciliana e la zona locrese, in particolare con Hipponion, subcolonia di Locri. Secondo il racconto riferito da Strabone ${ }^{88}$ prima di essere rapita da Ade, Core si era recata dalla Sicilia nei prati di Hipponion a raccogliere fiori, attratta dalla lussureggiante natura del luogo. Il racconto di Strabone suggerisce un legame con il choros dell'Inno omerico a Demetra: il rapimento di Core da parte di Ade avviene mentre la fanciulla stava danzando e raccogliendo fiori sul prato ${ }^{89}$. Questa versione del mito, che introduce Persefone come vergine nella cerchia delle fanciulle sue coetanee, secondo il tipo dei cori nell'ambito dei riti di passaggio femminili sfocianti nel matrimonio ${ }^{90}$, trova riscontro in quella dell'Elena di Euripide che narra come Demetra cercasse Core, rapita mentre danzava in coro $^{91}$. L'evento potrebbe essere richiamato dalla scena del choros delle tre fanciulle raffigurate nei pinakes del V sec. a.C., rinvenuti nel Persephoneion locrese (fig. 38) ${ }^{92}$; scene di ratto e di danza rituale, che in qualche caso presentano anche

79 Diodoro Siculo, Biblioteca storica V 49.

80 Longo, Le avventure pastorali di Dafni e Cloe IV 40, 1.

81 Posidippo, Danzatrici fr. 28 Kassel-Austin, in Ateneo, I Deipnosofisti IX 377a-b.

82 Cfr. BELL 1981, p. 92-93.

83 Strabone, Geografia XIV 649-650 C.

84 Polluce, Onomasticon I 37.

85 Diodoro Siculo, Biblioteca storica V 4; Plutarco, Dione 56.

86 Una testimonianza della presenza della danza nella vicenda di Core è ad Eleusi dove c'era un pozzo chiamato kallichoron, «dalle belle danze», dove per la prima volta le donne di Eleusi avevano danzato e cantato in onore della dea (Apollodoro, Biblioteca I 5; Pausania, I 38, 6). MYLONAS 1961, p. 72-73; SCARPI 2002, p. 474, n. 27; SHAPIRO 2004, p. 331, n. 283.

87 Nilsson 1957, p. 358; ZANCANI MONTUORO 1960, p. 37-50; PiCCALUGA 1974, p. 37; CALAME 2001, p. 92; SCARPI 2002, p. 442; CÀSSOLA 2006, p. 467.

88 Strabone, VI 1, 5. Cfr. PICCALUGA 1966, p. 233; SCARPI 2002, p. 446.

89 Inno omerico a Demetra vv. 3-8; 417-428. Cfr. CALAME 2001, p. 35. Si vedano inoltre LONSDALE 1993, p. 265-266; BURKERT 2003, p. 315.

90 CALAME 2001, p. 92; SCARPI 2002, p. 442.

91 Euripide, Elena vv. 1313-1315.

92 Pinakes di Locri III, p. 794-808, n. 1-44. 
Angela Bellia, Triadi di suonatrici nella Sicilia e nella Calabria di età greca (IV-III sec. a.C.), in (éd.) S. HuYSECOM-HAXHI, A. Muller, Figurines grecques en contexte. Présence muette dans le sanctuaire, la tombe et la maison, Lille, Presses universitaires du Septentrion-Université de Lille 3, 2015, pp. 107-126.

una suonatrice di tympanon, sono rappresentate anche nei busti fittili di Core-Persefone del IV sec. a.C. trovati a Morgantina e a Siracusa ${ }^{93}$.

Alla presenza degli strumenti musicali nelle triadi di suonatrici è talvolta associata, oltre alla colomba (fig. 30) ${ }^{94}$, uccello connesso alla sfera nuziale, anche quella di un oggetto rotondo, forse una palla (fig. 9) ${ }^{95}$, che non può non richiamare la raffigurazione di una sfera tenuta dai personaggi femminili raffigurati nell'atto dello svelamento connessi all'ambito nuziale di Core-Persefone ${ }^{96}$. L'uso di dedicare la palla a titolo di aparché al momento delle nozze, talvolta con una ciocca di capelli ${ }^{97}$, con le bambole e con gli strumenti musicali e i giocattoli sonori, simboleggia il passaggio al nuovo status nel mondo degli adulti delle fanciulle ${ }^{98}$. Un esempio è offerto da un epigramma dell'Antologia Palatina che associa l'offerta prenuziale della reticella per i capelli e della palla a quella dei tympana da parte di Timareta ad Artemide Limnatis ${ }^{99}$. Va anche notato che la palla era un particolare oggetto sonoro. Il rinvenimento di sonagli a forma di palla dotati di foro al polo superiore e contenenti palline d'argilla all'interno è documentato in Sicilia e in Calabria, sia nelle sepolture di fanciulli sia nei santuari delle divinità femminili protettrici delle nozze ${ }^{100}$. Il suono di questi crepitacoli, così come quello degli altri strumenti a percussione, come i kymbala e tympana, adatti a fornire il ritmo e ad accompagnare la danza, erano ritenuti in grado di stimolare la fecondità e usati per propiziare la fertilità e le nozze ${ }^{101}$.

Dallo studio emergono dati significativi che richiederanno approfondimenti interdisciplinari; alcuni meritano di essere qui accennati. Da un lato, si pone il problema della funzione delle triadi e del significato della dedica di questi fittili in specifici contesti. Se la presenza della musica emerge con ampiezza dalla documentazione archeologica, essa ricorre invece meno frequentemente nelle fonti scritte. Tuttavia, le informazioni fornite dai testi sottolineano l'esigenza di approfondire il ruolo della musica e della sfera sonora nell'ambito delle feste legate alle nozze di Core-Persefone. La loro celebrazione potrebbe essere in rapporto anche con gli eventi politici che interessarono la Sicilia e la Calabria ed in particolare con le nozze di Dionigi I con una donna siracusana e una locrese, avvenute, quasi contemporaneamente, nella prima decade del IV sec. a.C. ${ }^{102}$. Resta da accertare l'eventuale relazione simbolica tra questo evento, la musica per celebrarlo e la produzione delle triadi la cui diffusione, quantità,

93 Bell 1981, p. 140-141, n. 106c, tav. XXVIII. Cfr. PAUTASSO 2007, p. 216, nota 5; Portale 2008, p. 25 e p. 47.

94 PANVINI 2000, p. 30, n. 32; BELLIA 2009, p. 51, n. 64.

95 SARDELla-VANARIA 2000, p. 100; RAFFIOTTA 2007, p. 107, n. 151, fig. 29; SiRACUSANO 1996, p. 29, tav. XLII, 4; BELLIA 2006, p. 93-95, n. 77; RAFFIOTTA 2009, p. 121-122, fig. 34; BELLIA 2009, p. 103, nota 228 .

96 PAUTASSO 2008, p. 286, fig. 2.

97 Per il rito prenuziale del taglio dei capelli, cfr. DEUSSEN 1973, p. 125-133; CALAME 2001, p. 106-107; SABETAI 2008, p. 291-293.

98 L'offerta della palla e di una ciocca di capelli è raffigurata negli specchi di bronzo locresi. Si veda ARIAS 1977, p. 548-549.

99 Antologia Palatina VI 280. Cfr. TORELli 1977, p. 166; CALAME 2008, p. 37.

100 Bellia 2010, p. 82, fig. 6; Bellia 2012, c.s.

101 Per il potere apotropaico del suono e del «rumore» durante i riti nuziali, si veda OAKLEY-SINOS 1993, p. 26.

102 BRUNo SunZERI 2001, p. 359-369. Per la produzione di particolari raffigurazioni fittili nel periodo dionigiano, si vedano UHLENBROCK 2001, p. 319-335; PAUTASSO 2008, p. 285-291. 
Angela Bellia, Triadi di suonatrici nella Sicilia e nella Calabria di età greca (IV-III sec. a.C.), in (éd.) S. HUYSECOM-HAXHI, A. MULLER, Figurines grecques en contexte. Présence muette dans le sanctuaire, la tombe et la maison, Lille, Presses universitaires du Septentrion-Université de Lille 3, 2015, pp. 107-126.

cronologia e significato, nonché originalità iconografica, arricchisce notevolmente la documentazione delle testimonianze di interesse musicale finora note nel mondo greco. 
Angela Bellia, Triadi di suonatrici nella Sicilia e nella Calabria di età greca (IV-III sec. a.C.), in (éd.) S. HuYSECOM-HAXHI, A. MULleR, Figurines grecques en contexte. Présence muette dans le sanctuaire, la tombe et la maison, Lille, Presses universitaires du Septentrion-Université de Lille 3, 2015, pp. 107-126.

\section{BIBLIOGRAFIA}

ABRUZZESE CALABRESE 1996

ADAMESTEANU 1958a

ADAMESTEANU 1958b

ALBERTOCCHI 2004

AMANDRY 1984

ANGELINI 1994

ARIAS 1977

BAINES 1995

BARRA BAGNASCO 1986

BÉLIS 1984

BELL 1977

BELL 1981

BELL 1987

BELLIA 2005

BELLIA 2006

BELLIA 2006-2010

BELLIA 2009

BELLIA 2010

BELLIA 2012a

BELLIA 2012b

BERNABÒ BREA 1956

BREITENSTEIN 1945

BRUNO SUNZERI 2001

BUNDRICK 2005

BURKERT 2003
G. ABrUZZESE CAlABReSE, « Taranto », dans LiPPOLIS 1996, p. 189205

D. Adamesteanu, « Butera: Piano della Fiera, Consi e Fontana Calda. Fontana Calda. Scoperta della stipe votiva di un santuario campestre », MonAnt 44 (1958), col. 205-672

D. ADAMESTEANU, « Scavi e scoperte dal 1951 al 1957 nella provincia di Caltanissetta », NSc 12 (1958), p. 288-408

M. ALBERTOCCHI, Athana Lindia. Le statuette siceliote con pettorali di età arcaica e classica, RdA Suppl 28 (2004)

P. AMANDRY, « Le culte des Nymphes et de Pan à l'Antre corycien », dans L'antre corycien, BCH Suppl 9 (1984), p. 395-425.

F. ANGELINI, « Culti delle fonti », Mythos 6 (1994), p. 25-41

P.E. ARIAS, « L'arte locrese nelle sue principali manifestazioni artigianali. Terrecotte, bronzi, vasi, arti minori, in Locri Epizefirii », dans Locri Epizefirii (Colloque, Tarente, oct. 1976) (1977), p. 479579 .

A. BAINES, Storia degli strumenti musicali (1995) (titre original Musical Instruments through the Age [1969])

M. BARRA BAGNASCO, « La coroplastica votiva », dans LIPPOLIS 1996, p. 181-206.

A. BÉLIS, « Auloi grecs du Louvre », BCH 108 (1984), p. 111-122

M. BELL, « Le terrecotte votive del culto di Persefone a Morgantina », dans Il tempio greco in Sicilia. Architettura e culti (Colloque, Syracuse, nov. 1976), CronA 16 (1977), p. 140-147

M. Bell III, The Terracottas, Morgantina Studies 1 (1981)

M. BELL, « La fontana ellenistica di Morgantina », QuadAMess 2 (1986-1987), p. 111-124

A. Bellia, « Coroplastica con raffigurazioni musicali della Sicilia Greca », Aulos. Studi e ricerche di Archeologia musicale della Sicilia e del Mediterraneo 1 (2005), p. 49-63

A. BELLIA, Le raffigurazioni musicali nella coroplastica della Sicilia greca (VI-III sec. a.C.), Tesi di Dottorato, Università di Bologna (2006)

A. BELLIA, « Mito e rito nelle raffigurazioni musicali dei pinakes di Lipari », Imago Musicae 23 (2010), p. 11-24

A. Bellia, « Coroplastica con raffigurazioni musicali nella Sicilia greca (VI-III sec. a.C.) », Biblioteca di Sicilia Antiqua 3 (2009)

A. BELLIA, «Considerazioni sugli strumenti musicali di età arcaica, classica ed ellenistica nell'Italia meridionale e in Sicilia », SicAnt 7 (2010), p. 79-118

A. BELLIA, Il canto delle vergini locresi (2012)

A. BELLIA, Strumenti musicali e oggetti sonori nell'Italia meridionale e in Sicilia (2012)

L. BERNABÒ BREA, Akrai (1956)

N. Breitenstein, « Analecta Acragantina », AA 16 (1945), p. 8-153

G. BRUNO SUNZERI, « Matrimoni alla corte dei Dionisî », dans

N. Bonacasa, L. BracCESI, E. De Miro (éds), La Sicilia dei due Dionisî (1999), p. 359-369

S.D. BUNDRICK, Music and Image in Classical Athens (2005)

W. BURKERT, La religione Greca (2003) 
Angela Bellia, Triadi di suonatrici nella Sicilia e nella Calabria di età greca (IV-III sec. a.C.), in (éd.) S. HuYSECOM-HAXHI, A. Muller, Figurines grecques en contexte. Présence muette dans le sanctuaire, la tombe et la maison, Lille, Presses universitaires du Septentrion-Université de Lille 3, 2015, pp. 107-126.

Calame 2001

CALAME 2006

CALAME 2008

CALAme 2011

CANFORA 2001

CARRETTONI 1959

CÀsSOLA 2006

DE CESARE 2001

DE CESARE 2005

DE CESARE 2008

CIACERI 2004

CIPRIANI 1989

DEUSSEN 1973

FRASCA 1995

GENTILI 1995

GRASSO 1989

GUIZZI-STAITI 1992-1995

GUZZONE 2003

HINZ 1998

ISLER 1992

JACQUET RIMASSA 1999

KEKULÉ 1884

KOLLER 1995

LAGONA 1962

LAMAGNA 1994
C. CAlAmE, Choruses of Young Women in Ancient Greece (2001)

C. CALAME, L'amore in Grecia (2006)

C. CALAME, Iniziazioni femminili spartane, dans G. ARRIGONI (éd.), Le donne in Grecia (1985), p. 33-54

C. CALAME, Poetiche dei miti nella Grecia antica (2011)

L. CANFORA (éd.), Ateneo, I Deipnosofisti (2001)

G. CAREtTONI, « Tusa (Messina). Scavi di Halaesa », NSc 13 (1959), p. 293-349

F. CÀssola (éd.), Inni omerici (1994)

M. DE CESARE, « Immagine divina, mito e pratica rituale nella pittura vascolare greca. A proposito del cratere a calice siceliota di Siracusa con il mito delle Pretidi », MEFRA 113 (2001), p. 383-399

M. DE CESARE (éd.), CVA Italia. Museo Archeologico Regionale di Agrigento I (2005)

M. DE CESARE, « Terrecotte figurate », dans R. CAMERATA SCOVAZZO (éd.), Segesta III. Il sistema difensivo di Porta di Valle (scavi 19901993 (2008), p. 637-644.

E. CIACERI, Culti e miti nella storia dell'antica Sicilia ${ }^{3}$ (2004)

M. CIPRIANI, « San Nicola di Albanella. Scavo di un santuario campestre nel territorio di Posidonia-Paestum », dans M. TORELLI, A. Comella (éd.), Corpus delle stipi votive in Italia IV. Regio III, 1, (1989), p. 17-159

P. DEUSSEN, « The Nuptial Theme of Centuripe Vases », OpRom 9 (1973), p. 125-133

M. FRASCA, « Leontini. Piccola coroplastica ellenistica da una abitazione rupestre di contrada Crocefisso », $B d A 91$ (1995), p. 1-24

B. GENTILI, Poesia e pubblico nella Grecia antica ${ }^{3}$ (1995)

L. GRASSO, « Caracausi. Un insediamento rupestre nel territorio di Lentini. Coroplastica », CronA 28 (1989), p. 21-27

F. GUIZZI, N. STAITI, « Mania e musica nella pittura vascolare apula », Imago Musicae 9-12 (1992-1995), p. 43-90

C. Guzzone, « La stipe o deposito votivo di Fontana Calda », dans

R. PANVINI (éd.), Butera dalla preistoria all'età medievale (2003), p. $121-131$

V. HINZ, Der Kult von Demeter und Kore auf Sizilien und in der Magna Graecia, Palilia 4 (1998)

H.P. ISLER, « Monte Iato: la ventunesima campagna di scavo », SicA $25(1992) \mathrm{n}^{\text {os }} 78-79$, p. 7-43

P. JACQUET RIMASSA, « Les représentations de la musique, divertissement du symposion grec, dans les céramiques attique et italiote », REA 101 (1999), p. 37-63

R. KEKULÉ VON STRADONITZ, Die Terrakotten von Sizilien (Die antiken Terrakotten II) (1884)

H. Koller, « Ninfe, Muse, Sirene », dans D. RestAni (éd.), Musica e mito nella Grecia antica (1995), p. 97-107

S. LAGONA, « Rappresentazioni multiple di divinità femminili », CronA 1 (1962), p. 28-35

G. LAMAGNA, « Adrano (Catania). Contrade Difesa, Giobbe, Zaccani. Le recenti campagne di scavo nella città dionigiana di Adranon », $B A$ 28-30 (1994), p. 173-184 
Angela Bellia, Triadi di suonatrici nella Sicilia e nella Calabria di età greca (IV-III sec. a.C.), in (éd.) S. HuYSECOM-HAXHI, A. MULleR, Figurines grecques en contexte. Présence muette dans le sanctuaire, la tombe et la maison, Lille, Presses universitaires du Septentrion-Université de Lille 3, 2015, pp. 107-126.

LAMAGNA 1997-1998

LARSON 2001

LAWLER 1943

LIPPOLIS 1996

LONSDALE 1993

LORENZ 1864

MERKER 2000

MERTENS HORN 1991

MÜLLER 1915

MUTH 1954

MYLONAS 1961

NILSSON 1957

OAKLEY 2004

OAKLEY, SINOS 1993

OLIVIERI 1921

ORLANDINI 1960

ORLANDINI 1968

ORSI 1900

PACE 1945

PAGE 1955

PANVINI 1998

PANVINI 2000

PAPADOPOULOU 2004

PAQUETTE 1984

PAUTASSO 2007

PAUTASSO 2008

PicCALUGA 1966
G. LAMAGNA, «Alcuni dati sulle ultime campagne di scavo ad Adranon », Kokalos 43-44 (1997-1998), n² 2, p. 71-81

J. LARSON, Greek Nymphs. Myth, Cult, Lore (2001)

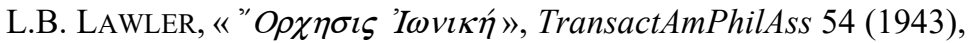
p. $60-71$

E. LiPPOLIS (éd.), I Greci in Occidente. Arte e artigianato in Magna Grecia (1996)

S.H. LONSDALE, Dance and Ritual in Greek Religion (1993)

A.O.F. LORENZ (éd.), Leben und Schriften des Koers Epicharmos: nebst einer Fragmentensammlung (1864)

G.S. MERKER, The Sanctuary of Demeter and Kore. Terracotta Figurines of the Classical, Hellenistic, and Roman Periods, Corinth 18.4 (2000)

M. MERTENS HoRn, « Una "nuova antefissa" a testa femminile da Akrai ed alcune considerazioni sul culto delle Ninfe in Sicilia », $B d A$ 76 (1991), no 66, p. 9-28.

V.K. Müller, Der Polos. Die griechische Götterkrone (1915)

R. MUTH, «'Hymenaios' und 'Epithalamion' », WSt 67 (1954), p. 545

G.E. MylonAS, Eleusis and the Eleusinian Mysteries (1961)

M.P. NILSSON, Griechische Feste von religiöser Bedeutung mit Ausschluss der Attischen (1957)

J.H. OAKLEY, « 4b Dance: Wedding Dance », ThesCRA 2 (2004), p. $312-314$

J.H. OAKLEY, R.H. SinOS, The Wedding in Ancient Athens (1993)

A. OLIVIERI (éd.), I frammenti della commedia dorica siciliana, (1921)

P. ORLANDINI, « Rinvenimenti nell'area dell'ospizio », NSc 14 (1960), p. 203-211

P. ORLANDINI, « Gela. Topografia dei santuari e documentazione archeologica dei culti », RIA 15 (1968), p. 20-66

P. ORSI, « Nuovo Artemision a Scala Greca », NSc 8 (1900), p. 353388

B. PACE, Arte e civiltà della Sicilia antica 3 (1945)

D.L. PAGE, Sappho and Alcaeus (1955)

R. PANVINI (éd.), Gela. Il Museo archeologico. Catalogo (1998)

R. PANVINI (éd), Gela: $i$ vasi ritrovati (sequestri 1970-2000) (2000)

Z. PAPADOPOUlOU, « 4b Dance: Artemis », ThesCRA 2 (2004), p. $325-330$

D. PAQUETTE, L'instrument de musique dans la céramique de la Grèce antique. Études d'organologie (1984)

A. PAutAsso, « Picturae in textili on Shouder Busts in Hellenistic Sicily? », dans C. GILlIS, M.-L.B. NosCH (éds), Ancient textiles. Production, Craft and Society (Colloque, Copenhague, mars 2003) (2007), p. 215-219

A. PAUTASSO, «Anakalypsis e Anakalypteria. Iconografie votive e culto di Persefone nella Sicilia dionigiana », dans C.A. DI STEFANO (éd.), Demetra. La divinità, i santuari, il culto, la leggenda (Colloque, Enna, juil. 2004), Biblioteca di Sicilia Antiqua 2 (2008), p. 285-291.

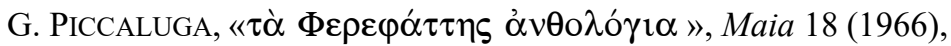
p. $233-253$ 
Angela Bellia, Triadi di suonatrici nella Sicilia e nella Calabria di età greca (IV-III sec. a.C.), in (éd.) S. HuYSECOM-HAXHI, A. MULleR, Figurines grecques en contexte. Présence muette dans le sanctuaire, la tombe et la maison, Lille, Presses universitaires du Septentrion-Université de Lille 3, 2015, pp. 107-126.

PICKARD-CAMBRIDGE 1962

Pinakes di Locri II

Pinakes di Locri III

PORTALE 2008

RAFFIOTTA 2007

RAFFIOTTA 2009

REICHERT-SÜDBECK 2000

RODRIGUEZ-GULLÉN 1996

SABETAI 2008

SACHS 1996

SACHS-HORNBOSTEL 2002

SALAPATA 2009

SARDELLA-VANARIA 2000

SCARPI 2002

SCHATKIN 1978

SCIBONA 1999

SÉCHAN 1930

SGUAITAMATTI 1984

SHAPIRO 2004

SIFAKIS 1971

SIRACUSANO 1996

TIBY 1993

TORELLI 1977
A.W. PICKARD-CAMBRIDGE, Dithyramb, Tragedy and Comedy ${ }^{2}$ (1962)

E. GRILlo, M. RubINICH, R. SchenAl PILEgGI, I pinakes di Locri Epizefiri. Musei di Reggio Calabria e di Locri II, AttiMemMagnaGr 2 (2000-2003), (2003)

F. Barello, M. Cardosa, E. Grillo, M. Rubinich, R. Schenal PILEGGI, I pinakes di Locri Epizefiri. Musei di Reggio Calabria e di Locri III, AttiMemMagnaGr 3 (2004-2007), (2007)

E.C. PORTALE, « Coroplastica votiva nella Sicilia di V-III secolo a.C.: la stipe votiva di Fontana Calda a Butera », SicAnt 5 (2008), p. 9-58

S. RAFFIOTTA, Terracotte figurate dal santuario di San Francesco Bisconti a Morgantina (2007)

S. RAFFIOTTA, « Nuove testimonianze del culto di Demetra e Persefone a Morgantina », dans G. GUZZETTA (éd.), Morgantina a cinquant'anni dall'inizio delle ricerche sistemantiche (Colloque, Aidone, dec. 2005) (2009), p. 105-139

P. REICHERT-SÜDBECK, Kulte von Korinth und Syrakus (2000)

L. Rodriguez, N. GuILlÉN (éds), Epicarmo de Siracusa. Testimonios y fragmentos (1996)

V. SABETAI, "Woman's Ritual Roles in the Cycle of Life », dans N. KALTSAS, A. SHAPIRO (éds), Worshipping Women. Ritual and Reality in Classical Athens (2008), p. 289-297

C. SACHS, Storia degli strumenti musicali (1996)

C. SACHS, E.M. VON HORNBOSTEL, « Systematik der Musikinstrumente, ein Versuch », ZEthn 46 (1914), p. 553-590

G. SALAPATA, « Female triads on Laconian Terracottas Plaques », BSA 104 (2009), p. 325-340

A. SARDELlA, M.G. VANARIA, « Le terrecotte figurate di soggetto sacrale del santuario dell'ex proprietà Maggiore di Lipari », dans L. BERnABÒ BREA, M. CAVALIER, Meligunis Lipára 10 (2000), p. 87 180

P. SCARPI (éd.), Le religioni dei misteri. I: Eleusi, dionisismo, orfismo (2002)

M.A. SCHATKIN, « Idiophones of the Ancient World. terminology, geographical distribution, functions ", JbAC 21 (1978), p. 142-172

G. SCIBONA, « La fortificazione greca e un nuovo quartiere di Messana in Età Classica », dans M.G. BACCI, G. TigANO (éds), Da Zancle a Messina. Un percorso archeologico attraverso gli scavi 2, (1999), p. 51-74

L. SÉCHAN, La danse grecque antique (1930)

M. SGUAITAMATTI, L'offrante de porcelet dans la coroplathie géléenne : étude typologique (1984)

H.A. SHAPIRO, « 4 b Dance: Demeter », ThesCRA 2 (2004), p. 331

G.M. SIFAKIS, Parabasis and Animal Choruses. A Contribution to the History of Attic Comedy (1971)

A. Siracusano, « L'Acropoli », dans A. CAlderone, M. CACCAMO Cattabiano, E. De Miro, A. Denti, A. Siracusano, Monte Saraceno di Ravanusa (1996), p. 7-40

O. TIBY, Antichi musicisti siciliani ${ }^{2}$ (1993)

M. TORELLI, « I culti in Locri », dans Locri Epizefirii (Colloque, Tarente, oct. 1976), (1977), p. 147-184 
Angela Bellia, Triadi di suonatrici nella Sicilia e nella Calabria di età greca (IV-III sec. a.C.), in (éd.) S. HUYSECOM-HAXHI, A. MULLER, Figurines grecques en contexte. Présence muette dans le sanctuaire, la tombe et la maison, Lille, Presses universitaires du Septentrion-Université de Lille 3, 2015, pp. 107-126.

TROPEA 1991

UHLENBROCK 2001

VANARIA 2000

WEST 2007

WILSON 2000

WILSON 2007

WINTER 1903

ZANCANI MONTUORO 1960

ZOPPI 2004

ZSCHÄTZSCH 2002
F. TropeA, « Suonatori », dans F. CostabILE (éd.), I ninfei di Locri Epizefiri: architettura, culti erotici, sacralità delle acque (1991), p. $180-189$

J.P. UHLENBROCK, « La coroplastica nella Sicilia orientale e meridionale nell'età dei due Dionisî: problemi di stile e di cronologia archeologica », dans N. BONACASA, L. BRACCESI, E. DE MIRO (éds), La Sicilia dei due Dionisî (Colloque, Agrigente, fév. 1999) (2001), p. $319-335$

M.G. VANARIA, « Su alcune terrecotte sporadiche da Valdichiesa di Leni (Salina) ", Nuovi studi di archeologia eoliana, Quaderni del Museo Archeologico Regionale Eoliano 3 (2000), p. 65-74

M.L. West, La musica greca antica (2007)

R.J.A. WILSON, « Aqueducts and Water Supply in Greek and Roman Sicily: the Present Status Quaestionis », dans G.C. M. JANSEN (éd.), Cura aquarum in Sicilia (2000), p. 5-36

P. WiLsON (éd.), The Greek Theatre and Festivals ( 2007)

F. WINTER, Die Typen der figürlichen Terrakotten I-II (Die antiken Terrakotten III.1-2) (1903)

P. ZANCANi MontuORO, « Il corredo della sposa », ArchCl 12 (1960), p. $37-50$

C. ZopPI, « Le fasi costruttive del cosiddetto santuario rupestre di San Biagio ad Agrigento: alcune osservazioni », SicAnt 1 (2004), p. 41-79

ZSCHÄTZSCH, Verwendung und Bedeutung griechischer Musikinstrumente in Mythos und Kult (2002) 
Angela Bellia, Triadi di suonatrici nella Sicilia e nella Calabria di età greca (IV-III sec. a.C.), in (éd.) S. HuYSECOM-HAXHI, A. Muller, Figurines grecques en contexte. Présence muette dans le sanctuaire, la tombe et la maison, Lille, Presses universitaires du Septentrion-Université de Lille 3, 2015, pp. 107-126.

\section{LÉGENDES}

Fig. 1. Diffusione delle triadi di suonatrici nella Sicilia e nella Calabria: 1. Adrano; 2. Agrigento; 3. Palazzolo Acreide; 4. Ciminna; 5. Gela; 6. Castel di Tusa; 7. Monte Iato; 8. Monte Saraceno; 9. Lentini; 10. Morgantina; 11. Butera; 12. Scornavacche; 13. Segesta; 14. Selinunte; 15. Solunto; 16. Siracusa; 17. Reggio Calabria; 18. Locri.

Fig. 2. Da Bellia 2009, p. 26, n. 16.

Fig. 3. Da Bellia 2009, p. 32-33, n. 28.

Fig. 4. Da BERnABÒ BrEA 1956, tav. XXXII, fig. 6.

Fig. 5. Da PACE 1945, p. 623, fig. 166;

Fig. 6. Da BELlia 2009, p. 47, n. 53.

Fig. 7. Da CARETTONI 1959, p. 314, fig. 22 a.

Fig. 8. Da ISLER 1992, p. 9, fig. 3.

Fig. 9. Da Siracusano 1996, p. 29, tav. XLII, 4.

Fig. 10. Da BELlia 2009, p. 61, n. 77.

Fig. 11. Da RAFFIOTTA 2007, p. 107, n. 151, fig. 29.

Fig. 12. Da ADAMESTEANU 1958a, col. 639, fig. 260.

Fig. 13. Da BELLIA 2009, p. 132, n. 332.

Fig. 14. Da DE CESARE 2008, p. 639, n. 11, tav. CVI.

Fig. 15. Da KeKUlÉ 1884, p. 14, fig. 20.

Fig. 16. Da BELLIA 2009, p. 138, n. 344.

Fig. 17. Da ORSI 1900, p. 383, fig. 29.

Fig. 18. Museo Archeologico Nazionale di Reggio Calabria. Inv. 359.

Fig. 19. Da TROPEA 1991, p. 181, n. 7, fig. 292.

Fig. 20. Da TROPEA 1991, p. 182, n. 9, fig. 294.

Fig. 21. Da BELliA 2012a, p. 118, fig. 36.

Fig. 22. Da SARDElla-VANARIa 2000, p. 95, tavv. II, 2.

Fig. 23. Da SARDELla-VANARIA 2000, p. 95, tav. X.

Fig. 24. Da LAMAGNA 1994, p. 78-79, tav. XLIX, fig. 1, 3;

Fig. 25. Da BELliA 2009, p. 25, n. 13.

Fig. 26. Da WINTER 1903, II, p. 140, fig. 8.

Fig. 27. Da GRASSO 1989, p. 23, n. 21, tav. V.

Fig. 28. Da BELLIA 2009, p. 25, n. 14.

Fig. 29. Da ADAMESTEANU 1958b, p. 311, n. 5.

Fig. 30. Da PANVINI 2000, p. 30, n. 32.

Fig. 31. Da FrASCA 1995, p. 10, n. 12, tav. II, d.

Fig. 32. Da ORLANDINI 1960, p. 58, fig. 41.

Fig. 33. Da BREITENSTEIN 1945, p. 136, fig. 51.

Fig. 34. Da BELlia 2009, p. 26, n. 15.

Fig. 35. Da BELlia 2009, p. 24, n. 8.

Fig. 36. Da BELliA 2009, p. 25, n. 11.

Fig. 37. Da FrASCA 1995, p. 10, n. 13, tav. II, c.

Fig. 38. Da Pinakes di Locri III, tav. CCII, b; fig. 65. 
Angela Bellia, Triadi di suonatrici nella Sicilia e nella Calabria di età greca (IV-III sec. a.C.), in (éd.) S. HuYSECOM-HAXHI, A. Muller, Figurines grecques en contexte. Présence muette dans le sanctuaire, la tombe et la maison, Lille, Presses universitaires du Septentrion-Université de Lille 3, 2015, pp. 107-126.

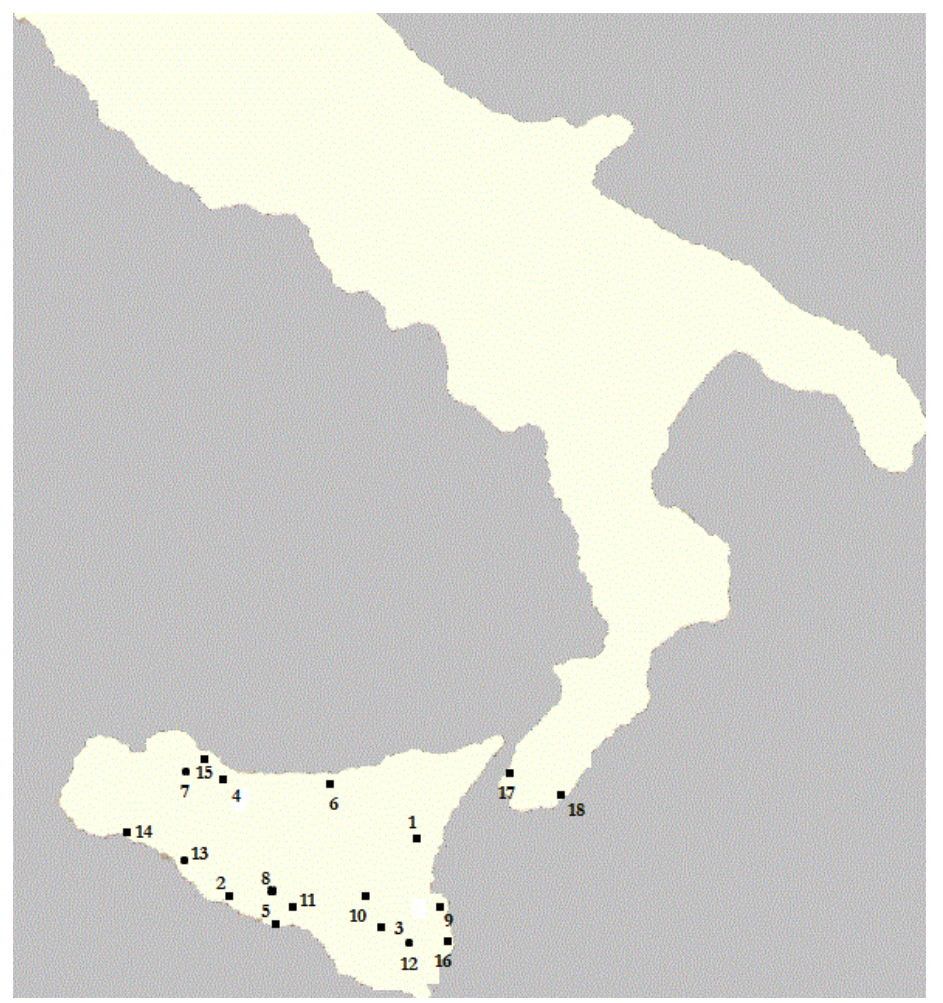

Fig. 1

1. Adrano; 2. Agrigento; 3. Palazzolo Acreide; 4. Ciminna; 5. Gela; 6. Castel di Tusa; 7. Monte Iato; 8. Monte Saraceno; 9. Lentini; 10. Morgantina; 11. Butera; 12. Scornavacche; 13. Segesta; 14. Selinunte; 15. Solunto; 16. Siracusa; 17. Reggio Calabria; 18. Locri.

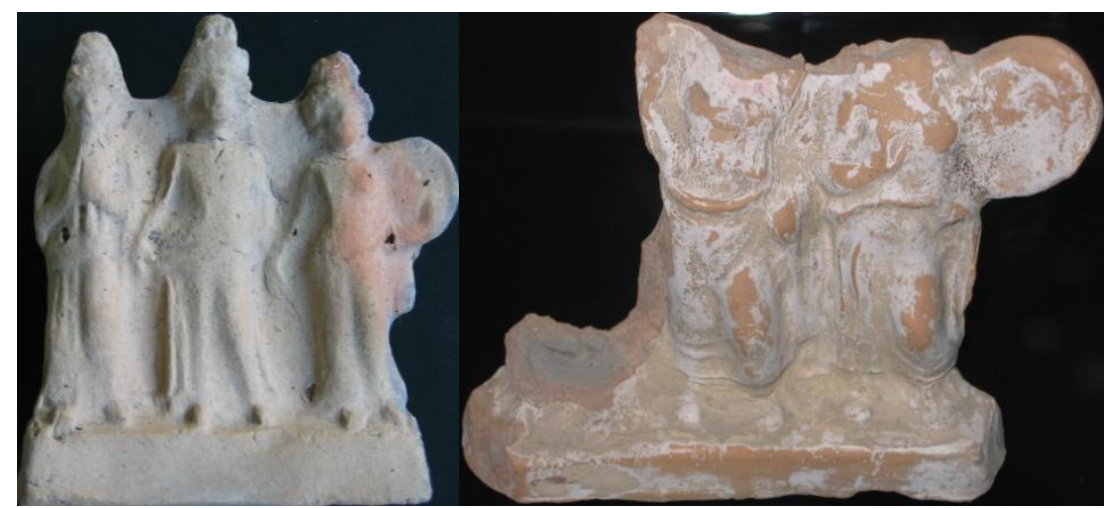

Fig. 2

Fig. 3

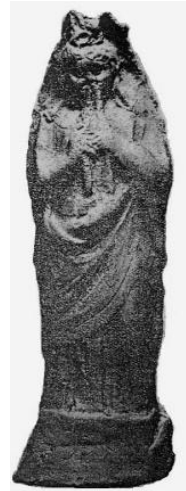

Fig. 4

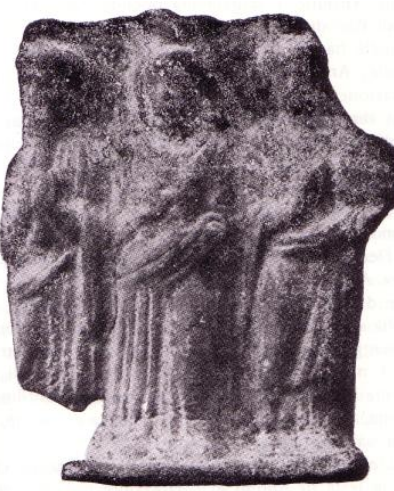

Fig. 5

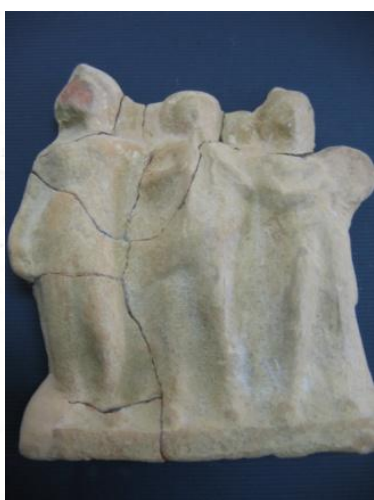

Fig. 6

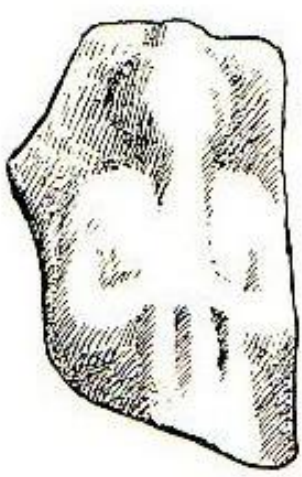

Fig. 7 
Angela Bellia, Triadi di suonatrici nella Sicilia e nella Calabria di età greca (IV-III sec. a.C.), in (éd.) S. HuYSECOM-HAXHI, A. Muller, Figurines grecques en contexte. Présence muette dans le sanctuaire, la tombe et la maison, Lille, Presses universitaires du Septentrion-Université de Lille 3, 2015, pp. 107-126.

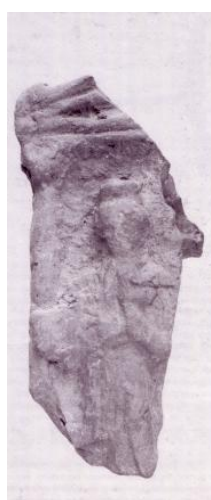

Fig. 8

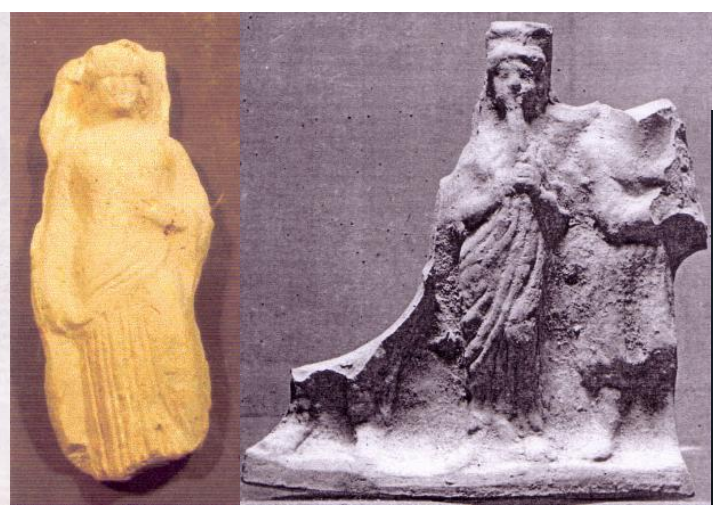

Fig. 9
Fig. 10

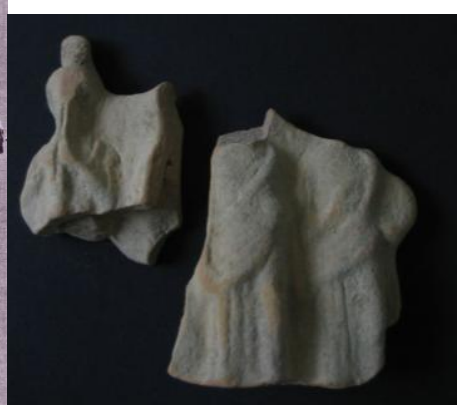

Fig. 11

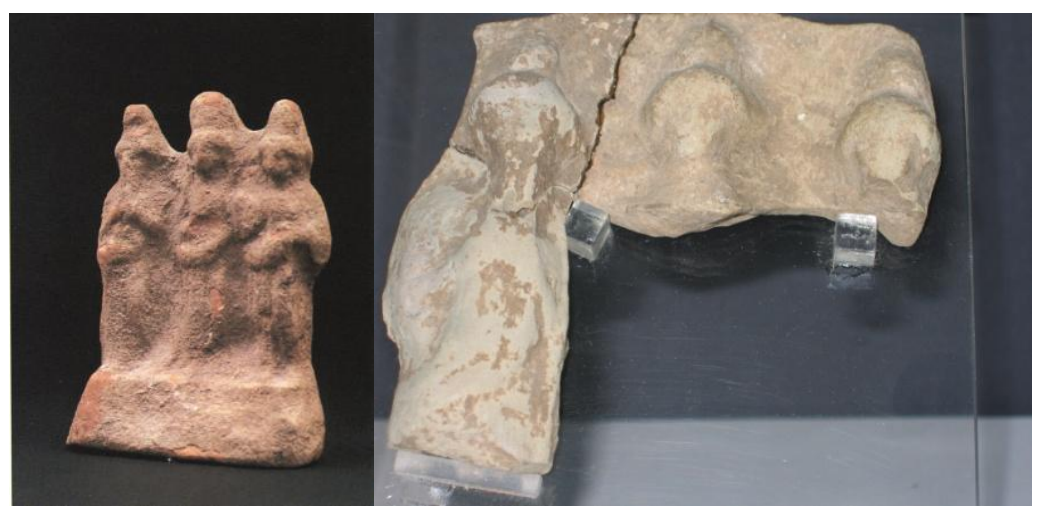

Fig. 12

Fig. 13

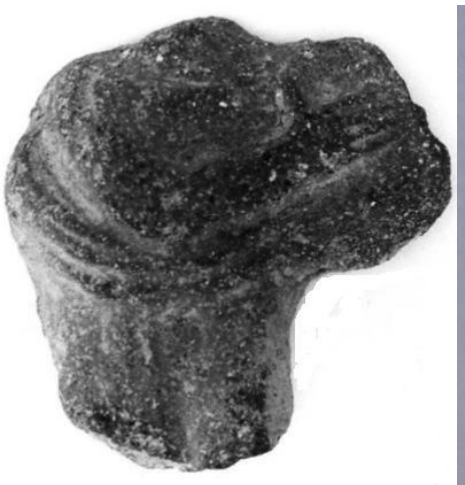

Fig. 14

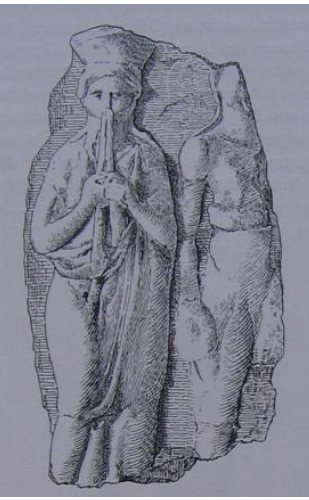

Fig. 15

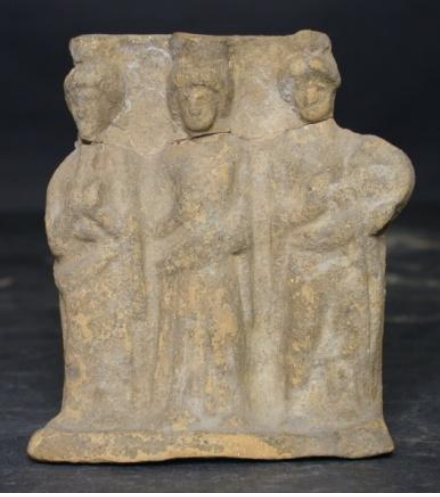

Fig. 16 
Angela Bellia, Triadi di suonatrici nella Sicilia e nella Calabria di età greca (IV-III sec. a.C.), in (éd.) S. HuYSECOM-HAXHI, A. Muller, Figurines grecques en contexte. Présence muette dans le sanctuaire, la tombe et la maison, Lille, Presses universitaires du Septentrion-Université de Lille 3, 2015, pp. 107-126.

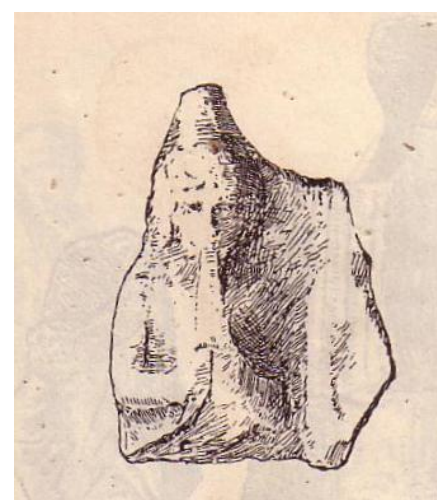

Fig. 17

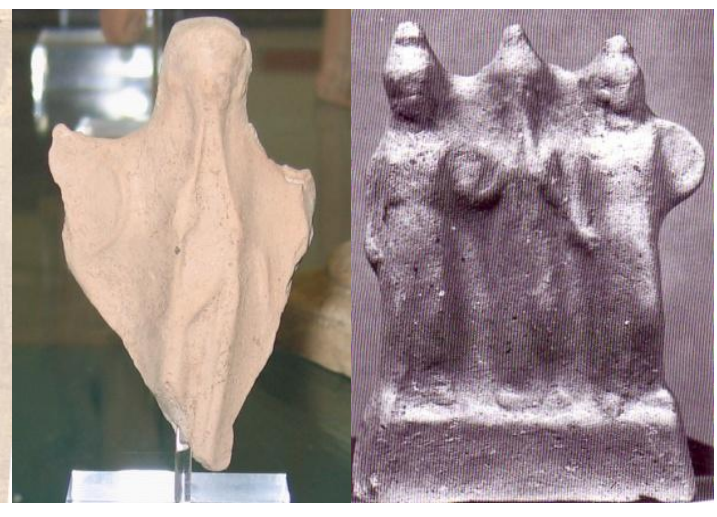

Fig. 18
Fig. 19

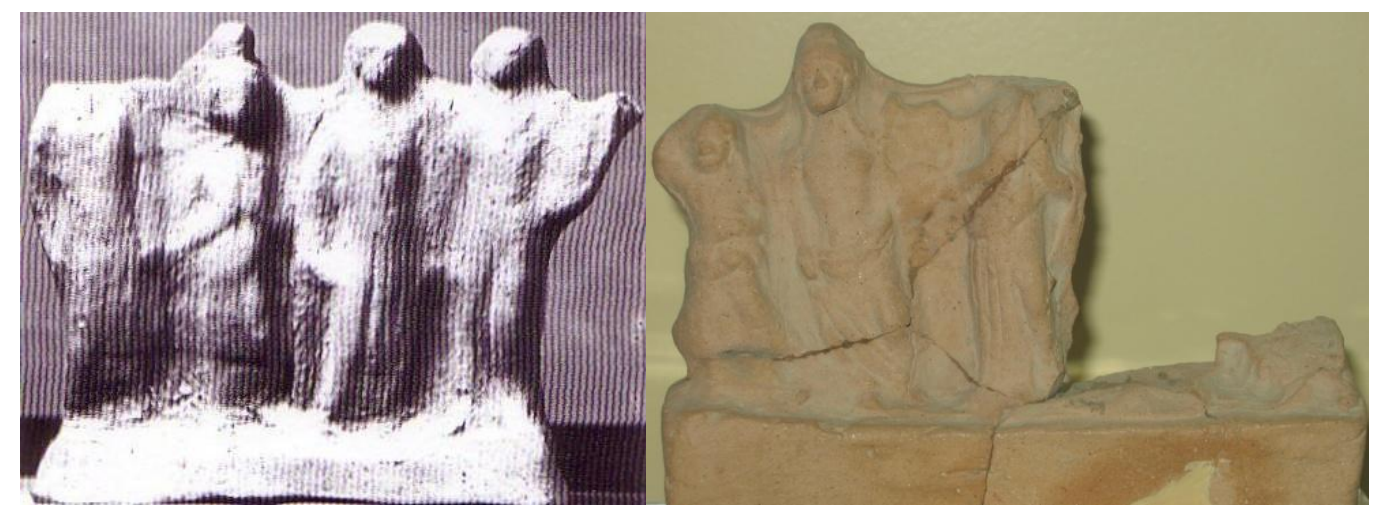

Fig. 20

Fig. 21

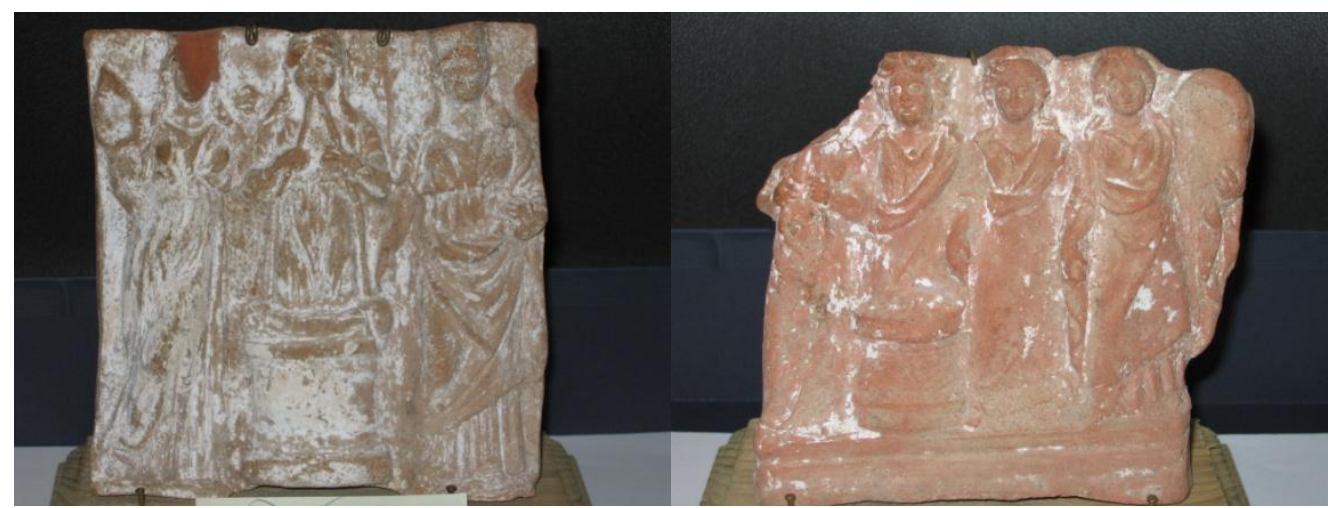

Fig. 22

Fig. 23 
Angela Bellia, Triadi di suonatrici nella Sicilia e nella Calabria di età greca (IV-III sec. a.C.), in (éd.) S. HuYSECOM-HAXHI, A. Muller, Figurines grecques en contexte. Présence muette dans le sanctuaire, la tombe et la maison, Lille, Presses universitaires du Septentrion-Université de Lille 3, 2015, pp. 107-126.

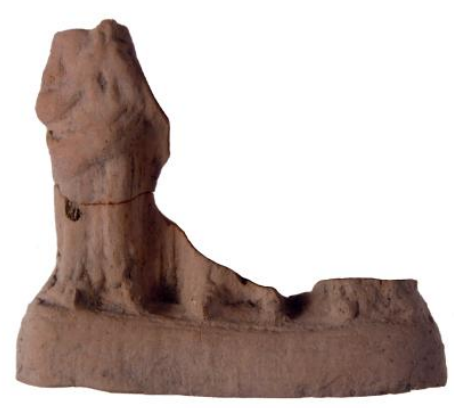

Fig. 24

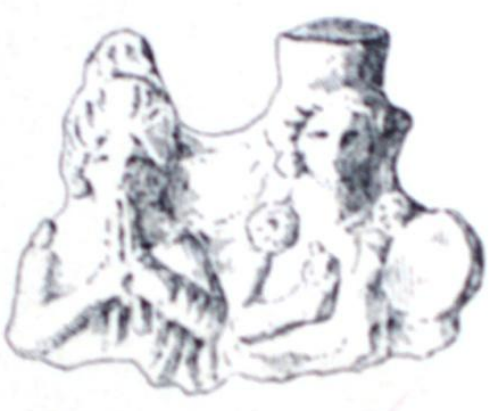

Fig. 26

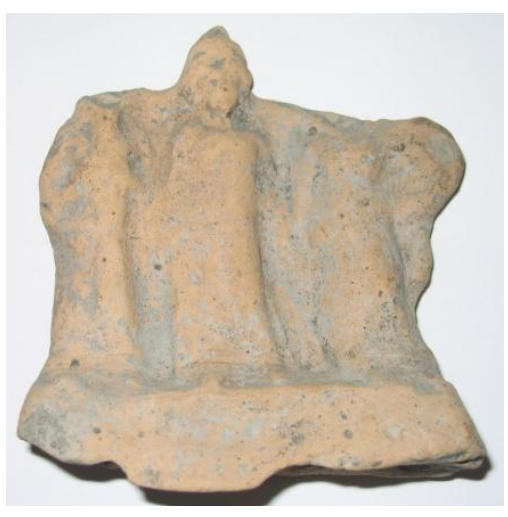

Fig. 25

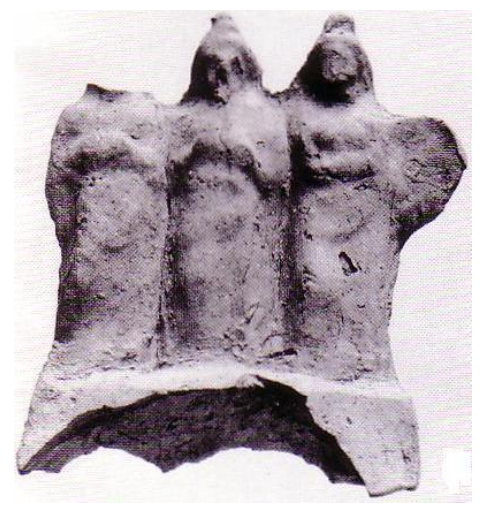

Fig. 27

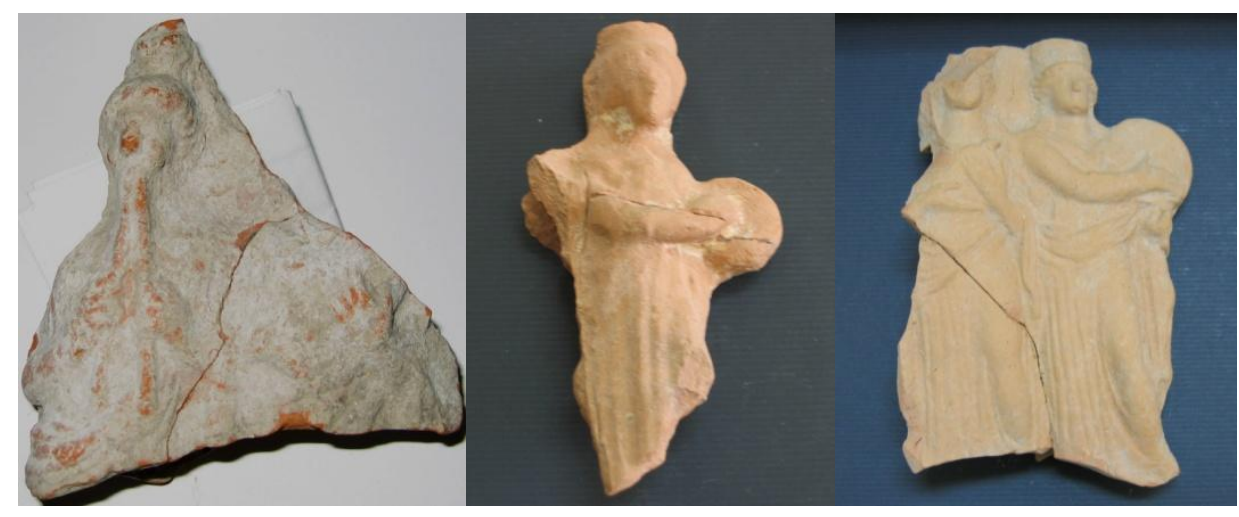

Fig. 28

Fig. 29

Fig. 30 
Angela Bellia, Triadi di suonatrici nella Sicilia e nella Calabria di età greca (IV-III sec. a.C.), in (éd.) S. HuYSECOM-HAXHI, A. Muller, Figurines grecques en contexte. Présence muette dans le sanctuaire, la tombe et la maison, Lille, Presses universitaires du Septentrion-Université de Lille 3, 2015, pp. 107-126.

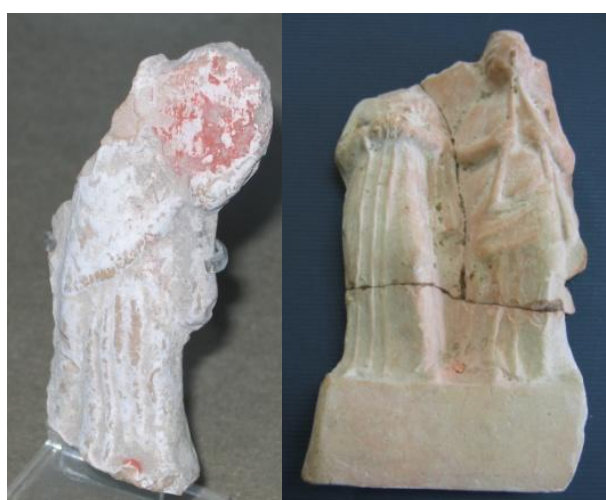

Fig. 31
Fig. 32

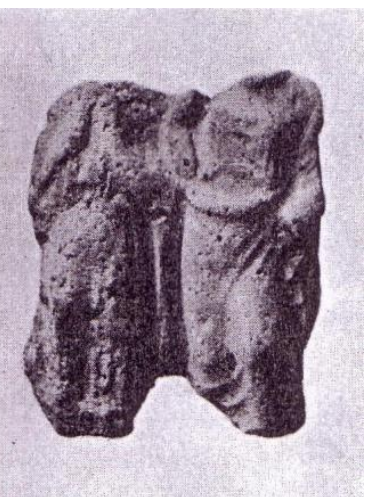

Fig. 33

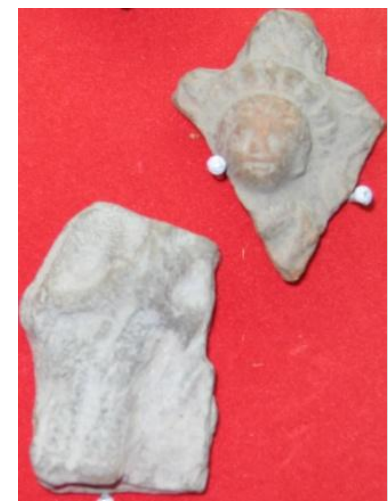

Fig. 34

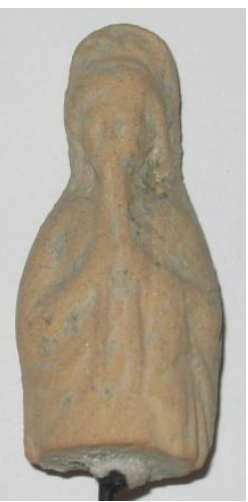

Fig. 35
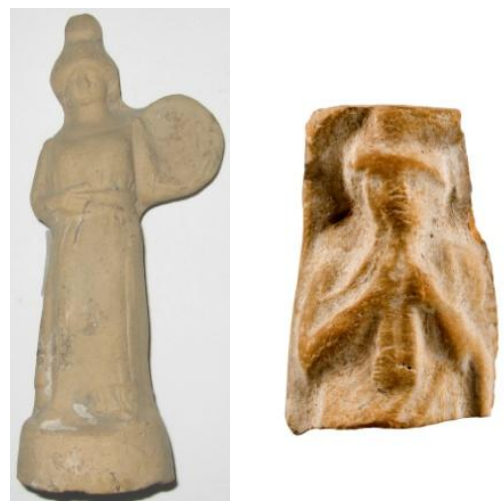

Fig. 36

Fig. 37
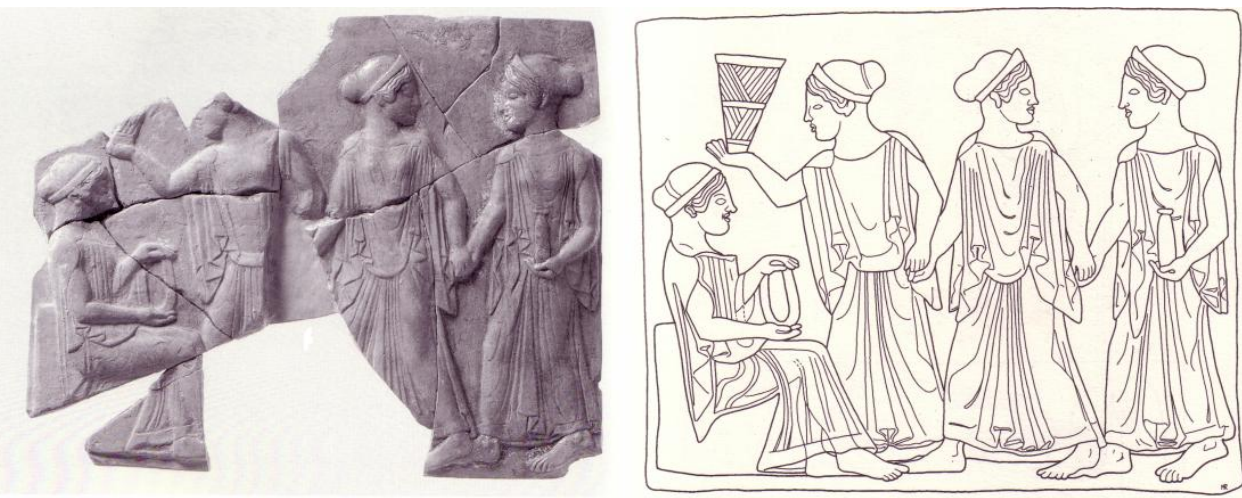

Fig. 38 